\title{
Prevalence of Trichomonas vaginalis in Pregnant Women in Zanjan, Northwest of Iran
}

\author{
Abbasali Nourian ${ }^{1, *}$, Nushin Shabani ${ }^{1}$, Asghar Fazaeli ${ }^{1}$, Seyed Noraddin Mousavinasab ${ }^{2}$ \\ ${ }^{1}$ Department of Medical Parasitology and Mycology, Zanjan University of Medical Sciences and Health Services, Zanjan, IR Iran \\ ${ }^{2}$ Department of Social Medicine, Faculty of Medicine, Zanjan University of Medical Sciences and Health Services, Zanjan, IR Iran \\ ${ }^{*}$ Corresponding author: Abbasali Nourian, Department of Medical Parasitology and Mycology, Zanjan University of Medical Sciences and Health Services, Zanjan, IR Iran. Tel: +98- \\ 9121417463, Fax:+241-4220678, E-mail: nourian@zums.ac.ir.
}

Received: July 15, 2012; Revised: September 24, 2012; Accepted: September 30, 2012

\begin{abstract}
Background: In the last two decades the incidence of sexually transmitted infections (STIs) are dramatically increased and remain a major public health problem in developing countries. Trichomoniasis is the most common non-viral sexually transmitted infection caused by Trichomonas vaginalis which is a flagellated protozoon associated with vaginitis, cervicitis and urethritis. Researchers believed that pregnancy is one of the influencing factors of the genital trichomoniasis in women.

Objectives: The aim of this study was to determine the prevalence of trichomoniasis in pregnant women in Zanjan, Iran.

Materials and Methods: In this cross-sectional study, 1000 pregnant women were examined for diagnosis of trichomoniasis based on direct microscopic examination and culture method. They referred to the treatment and health centers of Zanjan, Northwest Iran, Demographic and personal information of the subjects were collected and evaluated with questionnaires.

Results: Thirty-three out of one thousand examined individuals (3.3\%) presented vaginal infection with T. vaginalis. Infection in women with lower gestational age, higher mothers' age, higher parity and living in city were significantly associated with increased risk of vaginal trichomoniasis $(\mathrm{P}<0.05)$. Other variables were not significantly associated with parasite infection.

Conclusions: The present study found that the infection with T. vaginalis is a health problem in pregnant women correlated with some epidemiological variables and can be considered with microbiological screening tests during pregnancy.
\end{abstract}

Keywords: Trichomonas vaginalis; Diagnosis; Pregnant Women

\section{Background}

In the last two decades the incidence of sexually transmitted infections (STIs) is dramatically increased and remains a major public health problem in developing countries. Trichomoniasis is a sexually-transmitted disorder caused by Trichomonas vaginalis which is a flagellated protozoon. This parasitic infection may lead to vaginitis, cervicitis, pelvic inflammatory infection, and tubal infertility in females as well as urethritis and prostatitis in males $(1,2)$. In addition, it is also related to cervical cancer and an important complication in pregnancy which has been related with prematurity and low birth weight infants (3). Previous data have shown that more than 10 to $50 \%$ of women with trichomoniasis are asymptomatic and probably clinical manifestation will develop after half year of infection in $50 \%$ of those individuals $(3,4)$.

The most common symptoms are vulvar itching and malodorous vaginal discharge with yellow-green in color. Other symptoms that may appear include petechiae on the cervix and erythema of the vagina $(5,6)$. Typical clinical signs occur only in $12 \%$ of patients with T. vaginalis infection (7). Peak years of the prevalence for latter infection occur later in life among 20- to 46-year-olds (4). According to the world health organization data, the world wide prevalence of trichomoniasis would be 174 millions and to account for 10 to $25 \%$ of vaginal infections (1). Previous studies demonstrated that the prevalence of $T$. vaginalis infection was 1 to $42 \%$ in different geographical areas of Iran (8). The high rate (42\%) has probably been detected in special groups.

However, wet mount/potassium hydroxide $(\mathrm{KOH})$ is the conventional diagnostic method for non-viral disorder in the site of vagina which has been infected by T. vaginalis; the sensitivity of this method has been the case of debate, e.g. $58.8 \%$ in microscopy versus $95 \%$ in culture (9). Although, gold standard for diagnosis of trichomoniasis is liquid culture medium which has been considered the most accurate method, for early and correct identification novel molecular technique were crucially recommended. In the literature, limited data regarding trichomoniasis are available in Zanjan province. The only available report derived from a MD dissertation revealed

Implication for health policy/practice/research/medical education:

T. vaginalis is a health problem and crucially important in pregnant women, correlated with some demographic variables such as age, parity and residential place.

Copyright (C) 2013, Ahvaz JundishapurUniversity of Medical Sciences; Published by Kowsar Corp. This is an open-access article distributed under the terms of the Creative Commons Attribution License, which permits unrestricted use, distribution, and reproduction in any medium, provided the original work is properly cited. 
the infection rate of $6.25 \%$ in women referred to Vali-Asr Hospital of Zanjan (10); but there is no up to date information in this region. In additions, there is no information about prevalence of trichomoniasis in pregnant women.

\section{Objectives}

The purpose of this study was to determine the prevalence of trichomoniasis in pregnant women using two diagnostic methods including direct microscopic examination $(\mathrm{KOH})$ and in vitro culture.

\section{Materials and Methods}

In this cross-sectional study a total of 1000 vaginal samples from pregnant women who referred to obstetrics clinics at the Hospitals of Zanjan, were examined for detection of T. vaginalis using direct microscopic examination $(\mathrm{KOH})$ and Dorsset culture medium with observation of the parasite $(3,10)$ between July 2009 to June 2010. The present study was approved by the University Ethics Committee (code number: 882136) which provided written informed consent, subsequently attending underwent clinical examinations.

Gynecologist taken vaginal discharge samples from the vaginal fornix with 2 sterile wet swabs. Parasitological investigation for diagnosis of $T$. vaginalis of these samples included a microscopic examination with $0.5 \mathrm{ml}$ of normal saline buffer and a smear that was promptly prepared and observed under a light-field microscope. In addition, culturing of samples into the Dorsset liquid medium was performed. Briefly, this diphasic medium was prepared as following recipe; egg, distilled water (1/10 weight of broken egg), streptomycin (2 drops), starch powder $(0.5 \mathrm{~g})$ as a solid phase and ringer or saline buffer as a liquid phase. The second swab samples were inoculated and incubated at $37^{\circ} \mathrm{C}$ for more than 3 days as serially the liquid culture medium was observed daily with wet smears for investigation of T. vaginalis trophozoites. However, patients with blooding more than spotting were excluded from the study. Statistical analysis of data was performed using chi square test, Fisher's Exact test and Mann-Whitney Test.

\section{Results}

During one year follow up, one thousand $(n=1000)$ pregnant women were enrolled and agreed to participate in our study. The age distribution of those individuals adjusted in the present study was significantly different. Most of the patients were between 20 to 30 years old and the mean age of pregnant women was $26.4 \pm 6.2$ years. It was $58.4 \%$ and $41.6 \%$ for urban residents and rural residents, respectively. The portion of none educated subjects was12.4\% and the remaining showed different levels of education (Table 1). Most of the women (96.4\%) were housewife and only $3.6 \%$ had official job. The results have shown that, 33 out of 1000 pregnant women (3.3\%) were positive with $T$. vaginalis based on direct microscopic examination.

The parasite was consistently observed in 33 out of 1000 samples in Dorsset media culture for T. vaginalis . The results revealed a $100 \%$ agreement and correlation between these two performed methods in detection of T. vaginalis. Obtained data have demonstrated different characteristics of the patients with trichomoniasis. Figure 1 shows a significant variation between infected age groups, it means that the incidence of trichomoniasis in oldest individuals was higher than that of younger age groups $(\mathrm{P}=0.006)$ (Table 2). Gestational age in infected women $(36.5 \pm 4.5)$ at delivery time was lower than that of healthy women $(39 \pm 1.9)(\mathrm{P}=0.009)$. The infected women parity $(1.3 \pm 1.3)$ was higher than that of non- infected women $(0.7 \pm 0.9)(P=0.004)$.

As it is shown in Table 2, the prevalence of trichomoniasis in urban residents was high, comparing to infected women in the rural residents $(\mathrm{P}=0.04)$. However, no significant associations were observed between trichomoniasis and occupation $(\mathrm{P}=0.288$; Table 2$)$, income $(\mathrm{P}=$ 0.969; Table 2) and education ( $\mathrm{P}=0.059$; Table 1$)$.

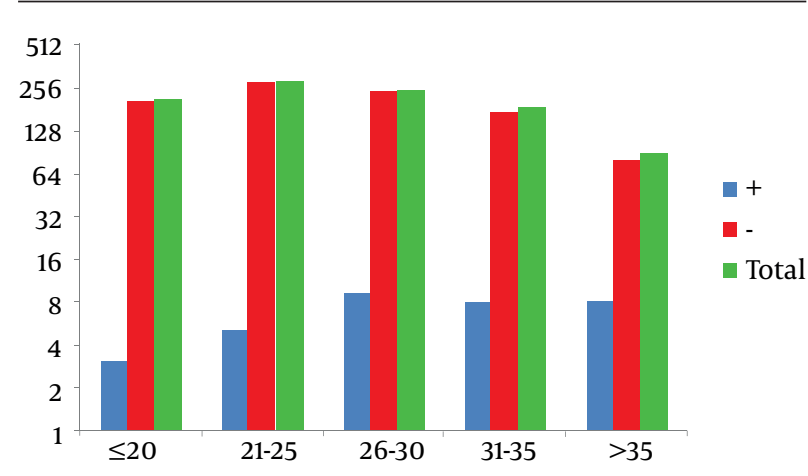

Figure 1. Frequency Distribution of T. vaginalis Infection in Different Age Groups of Pregnant Women in Zanjan $(\mathrm{P}=0.006)$.

Table 1. Frequency Distribution of T. vaginalis Infection Versus Education Level in Pregnant Women in Zanjan $(P=0.059)$

\begin{tabular}{llll}
\hline \multirow{2}{*}{ Education } & \multicolumn{2}{c}{ Trichomoniasis } & \multirow{2}{*}{ Total } \\
\cline { 2 - 3 } & Positive & Negative & \\
\hline No Education & $7(5.6 \%)$ & $117(94.4 \%)$ & $124(100 \%)$ \\
Under Diploma & $23(3.9 \%)$ & $574(96.1 \%)$ & $597(100 \%)$ \\
Diploma & $3(1.5 \%)$ & $194(98.5 \%)$ & $197(100 \%)$ \\
University & $0(0 \%)$ & $82(100 \%)$ & $82(100 \%)$ \\
Total & $33(3.3 \%)$ & $967(96.7 \%)$ & $1000(100 \%)$ \\
\hline
\end{tabular}


Nourian A et al.

Table 2. Frequency Distribution of T. vaginalis Infections Versus Residential Place, Occupation and Income in Pregnant Women in Zanjan.

\begin{tabular}{|c|c|c|c|c|c|}
\hline \multirow[t]{2}{*}{ Life Features } & & \multicolumn{2}{|c|}{ Trichomoniasis, No. (\%) } & \multirow[t]{2}{*}{ Total, No. (\%) } & \multirow[t]{2}{*}{ P value } \\
\hline & & Positive & Negative & & \\
\hline \multirow[t]{3}{*}{ Residential Place } & Urban & $25(4 / 3)$ & $559(95.7)$ & $584(100)$ & 0.04 \\
\hline & Rural & $8(1 / 9)$ & $408(98.1)$ & $416(100)$ & \\
\hline & Total & $33(3 / 3)$ & $967(96 / 7)$ & $1000(100)$ & \\
\hline \multirow[t]{3}{*}{ Occupation } & Housewife & $33(3.4)$ & $935(96.6)$ & $968(100)$ & 0.288 \\
\hline & Other & $0(0)$ & $32(100)$ & $32(100)$ & \\
\hline & Total & $33(3.3)$ & $967(96.7)$ & $1000(100)$ & \\
\hline \multirow[t]{4}{*}{ Income (US\$/mo) } & $<200$ & $16(3.2)$ & $490(96.8)$ & $506(100)$ & 0.969 \\
\hline & $200-300$ & $13(3.5)$ & $363(96.5)$ & $376(100)$ & \\
\hline & $>300$ & $4(3.4)$ & $114(96.6)$ & $118(100)$ & \\
\hline & Total & $33(3.3)$ & $967(96.7)$ & $1000(100)$ & \\
\hline
\end{tabular}

\section{Discussion}

Our results showed 3.3\% prevalence of $T$. vaginalis infection in pregnant women in Zanjan based on both methods of direct microscopy and in vitro culture. Higher age, higher parity age and urban residency were the studied variables that were significantly associated with higher rate of trichomoniasis. Differences in the infection rates were not significant among different education levels, income levels and occupation. Similar to other studies, increased rate of the infection was associated with raising age (11). This may be due to more sexual contact with increasing age. More frequent infection in women with higher parity also may be attributed to more changes in the genital tracts as one of the predisposing factors to the parasite growth.

The parasitic infection in urban resident women, comparing to rural resident women, was almost twice. This difference is difficult to interpret indeed and may require further investigation in terms of analysis of other related factors. A borderline of significant difference $(P=0.059)$ of the infection rate was seen among women with different education level with inverse association. Similar results were found in the United States (12); they found an inverse association between education and STIs, including trichomoniasis, in white race women but it was not significant in black women.

No significant association between occupation and trichomoniasis was seen, but this outcome cannot be reliable in this study, as most of the subjects had been housewife. In some studies, e.g. Sutcliffe et al. (13), low household income was correlated with $T$. vaginalis infection. This is not consistent with our results as we found no significant correlation; probably the household income itself and its differences among the study population are not significant and seem not effective on the infection rate. In epidemiological points of view, these sorts of variables may not influence the Trichomonas infection similarly in all communities. In both methods, in vitro culture and direct microscopic examination, all 33 samples were positive. Although direct method has lower sensitivity in comparison with other methods, including culture (14), the results are not consistent among different laboratories that may be affected by different factors such as the time of microscopic observation and possible intervals between sampling and observation times.

In the present study, we prepared and examined the wet mount directly after the swab sampling. This probably causes no missing of any positive sample with this method and indicates that the direct method is valuable if applied properly. Eastern European guideline for laboratory diagnosis of $T$. vaginalis encourages the utilization of wet mount of vaginal exudates as the first and more important option (15). It has been shown that the positive rates for trichomoniasis with different methods appear to have large confidence intervals because the prevalence of this infection is low in the studied population, in comparison with other genital infections, such as vulvovaginal candidiasis (22.4\%) and bacterial vaginitis (21.5\%) (16). Based on the published literature, sensitivity for direct examination is ranged from $35 \%$ to $80 \%(17,18)$, while in our study the sensitivity of microscopic examinations was the same as in vitro culture method.

According to the literature, no report about the infection in pregnant women in Iran was available but number of studies on non-pregnant women indicate almost similar prevalence, i.e. 2-3\% in Hamedan (19), 2.1-2.6\% in Yazd (20) and 3.3-3.6\% in Tehran (21). The infection rate in some of other countries was higher, e.g. 25\% in USA, 16-74\% in African countries (22), 3.4-3.8\% in Turkey and 5.1\% in India (22). High prevalence of the infection also observed in pregnant women of other countries; 20\% in America (23), 
17.3-18\% in Africa (11, 17), 12.1\% in HIV-1 infected pregnant women in Europe (24) and 9.8\% in immunocompetent pregnant women in Havana (25). We believed that the prevalence rates during pregnancy vary in the different published papers according to the populations examined.

Several studies have suggested that pregnant women infected with $T$. vaginalis may be at risk of any of adverse effects of the infection such as premature rupture of membranes, increased preterm delivery rates and lowbirth weight infants $(21,26-28)$. Some comparative studies showed that the pregnant women with trichomoniasis have $30 \%$ higher risk of delivering baby with low birth weight or delivering preterm than those without the infection (26). Also more prevalence of some STIs has been reported from pregnant women in some areas (11, 29); alteration of vaginal flora during pregnancy may be of relevant consecutive concerns (30). These indicate the importance of trichomoniasis in this group of women. Number of studies showed that the infection in pregnant women was up to $20 \%$ in the United States, $17.3 \%$ in Africa and $12.1 \%$ in Europe $(23,29,31)$. A study on immunocompetent pregnant women in Havana showed 9.8\% trichomoniasis (25).

Several factors like hygienic conditions, life styles, living environment and sexual and cultural behaviors can be responsible for these variable levels of infections; however, further investigation in different communities are required for such discussion. Meanwhile, the infection in pregnant women, may have effects on the pregnancy and fetuses, requires particular consideration. The present study found that the infection with T. vaginalis is a health problem and crucially important in pregnant women, correlated with some demographic variables such as age, parity and residential place, and might be considered as microbiological screening tests during pregnancy.

\section{Authors' Contribution}

Abbasali Nourian was the project executive manager. Nushin Shabani performed the practical part of the work as her MSc thesis. Asghar Fazaeli was the technical adviser. Seyed Noraddin Mousavinasab was the statistical adviser.

\section{Financial Disclosure}

This study was funded by the School of Medicine, Zanjan University of Medical Sciences and Health services, Zanjan, Iran. The authors report no potential conflicts of interest. The authors alone are responsible for the content and writing of the manuscript.

\section{Funding/Support}

Deputy for Research, ZUMS, Zanjan, Iran.

\section{References}

1. Faro S. Vaginitis. Differential diagnosis and management.. New York: Parthenon Publishing Group; 2004.

2. Edrisian Q, Rezaiian M, Qorbani M, Keshavarz H, Mohebali M. Medical porotozoology. 1 edTehran: Tehran uni pub; 2006.

3. Perazzi BE, Menghi CI, Coppolillo EF, Gatta C, Eliseth MC, de Torres RA, et al. Prevalence and comparison of diagnostic methods for Trichomonas vaginalis infection in pregnant women in $\mathrm{Ar}$ gentina. Korean J Parasitol. 2010;48(1):61-5.

4. Nelson AL, Woodward JA. Sexually transmitted diseases: A Practical Guide for Primary Care.New Jersey: Humana Press; 2006.

5. Saebi E. Parasitic diseases in Iran: protozoa. 4 edTehran: Aiij pub; 2005

6. Petrin D, Delgaty K, Bhatt R, Garber G. Clinical and microbiological aspects of Trichomonas vaginalis. Clin Microbiol Rev. 1998;11(2):300-17.

7. Fouts AC, Kraus SJ. Trichomonas vaginalis: reevaluation of its clinical presentation and laboratory diagnosis. I Infect Dis. 1980;141(2):137-143.

8. Valadkhani Z, Assmar M, Esfandiari B, Amirkhani A, Hassan N, Lotfi ML, et al. Trichomoniasis in Asymptomatic Patients. Iranian JPubl Health. 2008;37(3).

9. Wendel KA. Trichomoniasis: What's new? Curr Inf Dis Rep 2003;5(2):129-134.

10. Gharib Z. . Prevalence of Trichomonas vaginalis in pregnant women referred to Vali-e-Asr hospital, North-West of Iran, Zanjan.. ; 2004.

11. Kurewa NE, Mapingure MP, Munjoma MW, Chirenje MZ, Rusakaniko S, Stray-Pedersen B. The burden and risk factors of sexually transmitted infections and reproductive tract infections among pregnant women in Zimbabwe. BMC Infect Dis. 2010;10(1):127.

12. Annang L, Walsemann KM, Maitra D, Kerr JC. Does education matter? Examining racial differences in the association between education and STI diagnosis among black and white young adult females in the U.S. Public Health Rep. 2010;125 Suppl 4:110-21.

13. Sutcliffe S, Newman SB, Hardick A, Gaydos CA. Prevalence and correlates of Trichomonas vaginalis infection among female US federal prison inmates. Sex Transm Dis. 2010;37(9):585-90.

14. Huppert JS, Hesse E, Kim G, Kim M, Agreda P, Quinn N, et al. Adolescent women can perform a point-of-care test for trichomoniasis as accurately as clinicians. Sex Transm Infect. 2010;86(7):514-9.

15. Domeika M, Zhurauskaya L, Savicheva A, Frigo N, Sokolovskiy E, Hallen A, et al. Guidelines for the laboratory diagnosis of trichomoniasis in East European countries. J Eur Acad Dermatol Venereol. 2010;24(10):1125-34.

16. Coppolillo EF, Vay CA, Menghi CI, Eliseht MC, Gatta C, Mendez O, et al. Prevalencia de infecciones vaginales en embarazadas sintomáticas y asintomáticas. Rev EnferTract Gen Inf. 2007;1:17-22.

17. Patel SR, Wiese W, Patel SC, Ohl C, Byrd JC, Estrada CA. Systematic review of diagnostic tests for vaginal trichomoniasis. Infect Dis Obstet Gynecol. 2000;8(5-6):248-57.

18. Radonjic IV, Dzamic AM, Mitrovic SM, Arsic Arsenijevic VS, Popadic DM, Kranjcic Zec IF. Diagnosis of Trichomonas vaginalis infection: The sensitivities and specificities of microscopy, culture and PCR assay. Eur J Obstet Gynecol Reprod Biol. 2006;126(1):116-20.

19. Habibipour R, Amirkhani A, Matin Nya N. Rate of Trichomonas vaginalis infection in women referred to public security hospitals of Hamedan, 2004. Tabib-e-Shargh. 2006;8:245-251.

20. Etminan Rad S, Bokaii M. Investigation of Trichomoniasis infection in women referred to clinic of gynaecology and hygienic centers of Yazd, 2006. Sci J Health. 2007;2:14-20.

21. Cotch MF, Pastorek JG, 2nd, Nugent RP, Hillier SL, Gibbs RS, Martin DH, et al. Trichomonas vaginalis associated with low birth weight and preterm delivery. The Vaginal Infections and Prematurity Study Group. Sex Transm Dis. 1997;24(6):353-60.

22. Schwebke JR, Burgess D. Trichomoniasis. Clin Microbiol Rev 2004;17(4):794-803.

23. Garber GE. The laboratory diagnosis of Trichomonas vaginalis. 
Nourian A et al.

Can J Infect Dis Med Microbiol. 2005;16(1):35-8.

24. Landes M, Thorne C, Barlow P, Fiore S, Malyuta R, Martinelli P, et al. Prevalence of sexually transmitted infections in HIV-1 infected pregnant women in Europe. Eur JEpidemiol. 2007;22(12):925-36.

25. Fernandez Limia O, Lantero MI, Betancourt A, de Armas E, Villoch A. Prevalence of Candida albicans and Trichomonas vaginalis in pregnant women in Havana City by an immunologic latex agglutination test. Med Gen Med. 2004;6(4):50.

26. Riggs MA, Klebanoff MA. Treatment of vaginal infections to prevent preterm birth: a meta-analysis. Clin Obstet Gynecol. 2004;47(4):796-807.

27. Hay P, Czeizel AE. Asymptomatic trichomonas and candida colonization and pregnancy outcome. Best Pract Res Clin Obstet Gynaecol. 2007;21(3):403-9.

28. Hillier SL, Nugent RP, Eschenbach DA, Krohn MA, Gibbs RS, Martin $\mathrm{DH}$, et al. Association between bacterial vaginosis and preterm delivery of a low-birth-weight infant. The Vaginal Infections and Prematurity Study Group. N Engl J Med. 1995;333(26):1737-42.

29. Menendez C, Castellsague X, Renom M, Sacarlal J, Quinto L, Lloveras B, et al. Prevalence and risk factors of sexually transmitted infections and cervical neoplasia in women from a rural area of southern Mozambique. Infect Dis Obstet Gynecol. 2010;2010.

30. Gondo DC, Duarte MT, da Silva MG, de Lima Parada CM. Abnormal vaginal flora in low-risk pregnant women cared for by a public health service: prevalence and association with symptoms and findings from gynecological exams. Rev Lat Am Enfermagem. 2010;18(5):919-27.

31. Tann CJ, Mpairwe H, Morison L, Nassimu K, Hughes P, Omara M, et al. Lack of effectiveness of syndromic management in targeting vaginal infections in pregnancy in Entebbe, Uganda. Sex Transm Infect. 2006;82(4):285-9. 